\title{
Synchronous multiple primary gallbladder and gastric malignancies: Report of two cases and review of the literature
}

\author{
SHUO ZHOU ${ }^{1}$, ZHENG LU ${ }^{1}$, HUA WU ${ }^{1}$, CONG-YOU GU ${ }^{2}$, DENG-YONG ZHANG ${ }^{1}$, \\ WAN-LIANG SUN ${ }^{1}$, XIANG MA ${ }^{1}$ and HUI-CHUN LIU ${ }^{1}$ \\ Departments of ${ }^{1}$ Hepatobiliary Surgery and ${ }^{2}$ Pathology, The First Affiliated Hospital \\ of Bengbu Medical College, Bengbu, Anhui 233000, P.R. China
}

Received August 24, 2016; Accepted August 17, 2017

DOI: $10.3892 /$ mco.2017.1397

\begin{abstract}
Multiple primary malignancies (MPM) are rare. In particular, synchronous gallbladder and gastric malignancies are extremely rare, are associated with a concealed onset and atypical symptoms, and are highly likely to be overlooked or misdiagnosed. The clinical data of two patients with synchronous gallbladder and gastric malignancies are herein reported and integrated with the relevant literature to retrospectively analyze and summarize the pathogenesis and clinical characteristics of MPM. Case 1 was a male 46-year-old patient who underwent laparoscopic cholecystectomy, and succumbed to extensive tumor metastasis 2 months after the operation. Case 2 was an 80-year-old female patient who was treated with distal gastrectomy for gastric cancer, cholecystectomy, gastrojejunostomy and dissection of 5 suprapyloric, 6 subpyloric, 7 left gastric and 8 common hepatic artery lymph nodes, and succumbed to multiple organ failure induced by extensive tumor invasion within 1 week after the operation. Clinical physicians must pay closer attention to early symptoms of MPM in order to make an accurate diagnosis, perform timely radical surgical treatment and achieve favorable therapeutic outcomes, in terms of significantly increasing long-term patient survival rates.
\end{abstract}

\section{Introduction}

The term multiple primary malignancies (MPM) refers to concurrent or successively occurring $\geq 2$ independent primary malignancies, and may generally appear in one system organ, paired organs, or a single organ. Among reported MPM cases, $76 \%$ occurred in different sites, and the remaining $24 \%$ in the

Correspondence to: Dr Zheng Lu, Department of Hepatobiliary Surgery, The First Affiliated Hospital of Bengbu Medical College, 287 Chang Huai Road, Bengbu, Anhui 233000, P.R. China

E-mail: luzhengdr@163.com

Key words: multiple primary malignancies, gallbladder carcinoma, gastric carcinoma, pathogenesis, clinical characteristics, treatments same sites as the primary cancer (1). MPM is a rare condition with a low prevalence rate, but exhibiting a tendency for increased prevalence in recent years. In the literature, statistical data on MPM report a prevalence rate of $0.73-11.7 \%$ (2), with the differences attributed to the different researchers, diagnostic and treatment technologies, and different dietary and living conditions in different areas. Little is known on the pathogenic risk factors and the mechanism underlying the pathogenesis of MPM. In addition, due to its low incidence rate and insidious onset, MPM may be easily overlooked or misdiagnosed. In addition, there is currently no international consensus for the clinical diagnosis and treatment of MPM. A total of 2 cases with synchronous primary gallbladder and gastric malignancies are reported herein. In addition, the pathogenesis, clinical characteristics and diagnostic and treatment methods are integrated with the relevant literature.

\section{Case reports}

Case 1. A 46-year-old male patient was admitted with intermittent right upper abdominal pain and distension for 2 months, with reflux symptoms, poor appetite and a weight loss of $\sim 5 \mathrm{~kg}$. With respect to previous history, the patient had been diagnosed with hypertension 8 years prior, and had been routinely taking oral captopril. In addition, the patient had received a craniotomy after an accident 3 years prior. There was a history of epilepsy after the surgery and the patient received sodium valproate orally. The family history was not significant. On physical examination, the patient was conscious, with speech disfluency and tenderness in the upper right abdominal quadrant, with tympanitic sound on abdominal percussion. Furthermore, the blood pressure was $155 / 104 \mathrm{mmHg}$. Following laboratory examinations, the carcinoembryonic antigen (CEA) level was $8.79 \mathrm{ng} / \mathrm{ml}$ (normal range, $0-5 \mathrm{ng} / \mathrm{ml}$ ). The abdominal color Doppler ultrasound revealed that the gallbladder was sized $\sim 40 \times 80 \mathrm{~mm}$, with a wall thickness of $4 \mathrm{~mm}$ and coarse appearance. The transmission performance of the bile was fair and a substantial hyperechoic area $(14 \times 28 \mathrm{~mm})$ was identified in the middle of the gallbladder, with an irregular shape and a relatively wide basilar part. A computed tomography (CT) scan of the epigastrium revealed increased gallbladder volume, with a locally thickened wall and nodular high-density shadows in the cavity, with inten- 
sification on contrast-enhanced CT (Fig. 1A). As there were no surgical contraindications, laparoscopic cholecystectomy (LC) was conducted under general anesthesia. Intraoperative laparoscopic exploration revealed mild adhesion of the gallbladder and the omentum, a gallbladder size of $\sim 8 \times 4 \times 4 \mathrm{~cm}$, a common bile duct diameter of $5 \mathrm{~mm}$, without abnormalities of other organs. On postoperative examination, the thickness of the gallbladder wall was $4 \mathrm{~mm}$, with a gray-white broad-based polyp $(5 \times 5 \mathrm{~mm}$ ) attached to the wall in the caudomedial part of the gallbladder. Frozen section pathology revealed gallbladder adenocarcinoma. However, the patient and his family refused further surgical treatment against medical advice; thus, radical cholecystectomy was not performed. Postoperative pathological examination revealed grade II gallbladder adenocarcinoma, with coarse foci and hyperplasia of the mucosal surface (Fig. 1B). On the fourth postoperative day, the patient complained of poor appetite, and electronic gastroscopy and biopsy were performed. The mucous membrane of the gastric fundus was coarse with irregular ulcerations, hard and prone to bleeding. The expansion ratio of the gastric cavity was poor and the mucous membrane of the gastric antrum was coarse and edematous. Foci of poorly differentiated carcinoma of the gastric fundus, with a squamous epithelial component, were diagnosed histologically (Fig. 1C). The patient and his family refused further treatment and, after receiving adjuvant therapies (intravenous infusion of omeprazole to protect the gastric mucosa and monitoring of fluid balance to prevent electrolytic disorders), the patient was discharged 10 days later. The diagnosis upon discharge was i) primary gallbladder carcinoma, ii) primary invasive gastric carcinoma, iii) traumatic epilepsy and iv) grade II (high-risk) hypertension. The patient succumbed to extensive metastasis of the tumor 2 months after being discharged from the hospital.

Case 2. An 80-year-old female patient complained of dull pain in the upper right abdomen, aggravated pain during night-time, poor appetite and dry stools. There was no significant past medical or family history. Physical examination revealed no abnormalities. The laboratory examination revealed a CEA level of $4.06 \mathrm{ng} / \mathrm{ml}$ (normal range, $0-5 \mathrm{ng} / \mathrm{ml}$ ) and a carbohydrate antigen 19-9 level of $68.84 \mathrm{U} / \mathrm{ml}$ (normal range, 0-37 U/ml). The electrocardiogram revealed sinus rhythm with prolongation of the P-R interval ( $>0.21 \mathrm{~s}$ ), whereas there was reduced intensity of the first heart sound on auscultation of the cardiac apex. The color Doppler ultrasound of the epigastrium revealed a size of the fasting gallbladder of $\sim 82 \times 20 \mathrm{~m}$. The wall of the gallbladder was thickened and the echogenicity was high, with a local cauliflower-like appearance. A CT of the abdomen revealed a thickened gallbladder wall and a high-density shadow in the cavity of the gallbladder (10x10 mm), with an irregular shape. Contrast-enhanced CT revealed intensification (Fig. 2A). The patient underwent laparotomy 6 days after admission. On intraoperative exploration, the gallbladder was enlarged ( 8x6x6 mm), its consistency was hard, the diameters of cystic bile duct and the common bile duct were 6 and $\sim 20 \mathrm{~mm}$ respectively, and there was a hard mass $(\sim 10 \mathrm{~mm})$ in the caudomedial part of the gallbladder wall. There were tumor deposits of different sizes on the greater omentum and the peritoneum. There was an accessible hard mass $(\sim 6 \times 5 \mathrm{~cm})$ in the gastric antrum, which perforated the serosal layer. The suprapyloric, subpyloric, left gastric and common hepatic artery lymph nodes were enlarged and the common bile duct was dilated to $\sim 2 \mathrm{~cm}$. Distal gastrectomy for gastric cancer, cholecystectomy, and dissection of 5 suprapyloric, 6 subpyloric, 7 left gastric and 8 common hepatic artery lymph nodes were conducted. Routine postoperative pathology (Fig. 2B-D) revealed grade III gastric adenocarcinoma with local invasion. The tumor was located near the pylorus and invaded the submucosa and muscular layer; the serosa and lamina propria of the mucosa were also invaded by tumor cells. Intravascular cancer emboli were detected. The lymph nodes in close proximity to the mass were positive, but the proximal and distal resection margins were negative. The diagnosis was grade III adenocarcinoma of the gallbladder, with tumor invasion of all layers of the wall. The patient was discharged 12 days after the operation. The diagnosis on discharge was i) cancer of the gastric antrum and ii) gallbladder carcinoma. Within 1 week after the discharge, the patient succumbed to extensive tumor metastasis.

\section{Discussion}

Since MPM was first reported by Billroth et al in 1889, its prevalence rate has increased, with a peak age of onset at 50-70 years. In the USA, statistics on a total of 96,00000 patients who were diagnosed with tumors between 1975 and 2001, revealed that MPM patients accounted for $7.9 \%$ of all cancer cases. Among the MPM cases, $76 \%$ occurred in different sites, and the remaining $24 \%$ in the same sites as primary cancer (1). In the literature, statistical data on MPM report a prevalence rate of $0.73-11.7 \%$ (2), with the differences attributed to the different researchers, diagnostic and treatment technologies, and the different dietary and living conditions in different areas. However, according to statistics on autopsy cases by the Japanese Medical Center (3), the proportion of cases with double primary cancers in all tumor cases increased from $3.16 \%$ in 1980 to $7.49 \%$ in 1997 , and the proportion of triple primary cancers also increased from 0.2 to $1.33 \%$. Generally, MPM frequently appears in the digestive system. Common MPMs include gastric carcinoma with colorectal cancer, gastric carcinoma with renal cancer, lung cancer with esophageal cancer and gallbladder carcinoma, and gastric carcinoma with carcinoma of the sigmoid colon and others (3-6), among which double primary cancers are more common, while triple and higher order primary cancers are relatively rare.

The two cases reported herein are cases of synchronous primary gallbladder carcinoma and gastric cancer. As regards the pathogenesis of MPM of the digestive tract, it may be associated with multiple-spot cancerization and intracavitary implantation metastasis. The field cancerization theory presented by Slaughter et al (7) and Brown et al (8) may be a plausible explanation. Namely, mucosa covering a widespread area, rather than mucosal foci, is exposed to a carcinogen. For example, the digestive, respiratory and urinary systems are exposed to chronic stimulation by common carcinogens in the food and in the air, leading to cumulative gene alterations and continuous or discontinuous multiple independent foci of chronic inflammation; dysplasia and cancer foci form in the mucosa, and multiple primary cancers gradually develop. As regards the two cases presented herein, gallbladder and gastric 

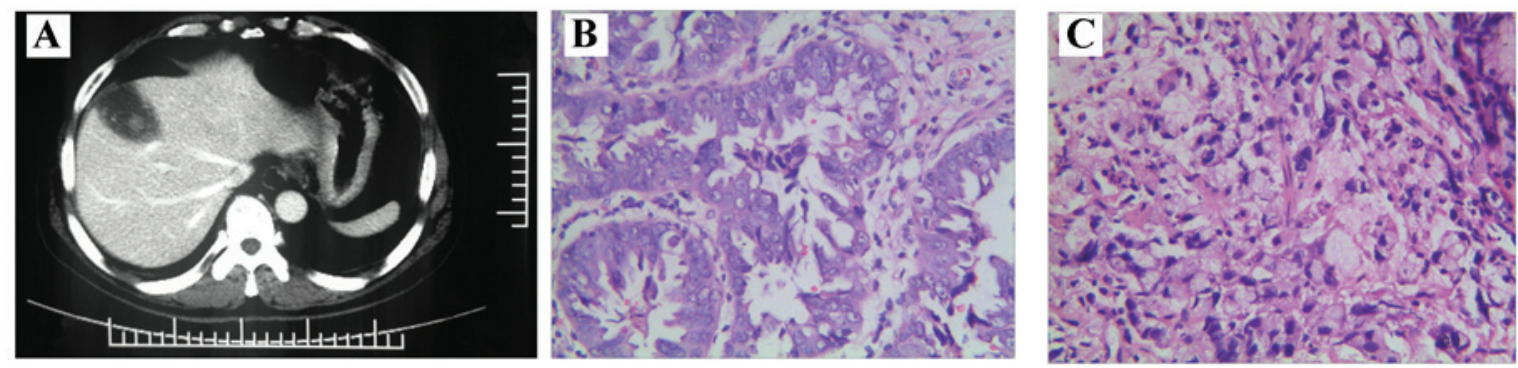

Figure 1. (A) Abdominal computed tomography scan showing a hyperechoic area located in the gallbladder. (B) Postoperative gallbladder pathology revealed gallbladder adenocarcinoma, with surface coarse foci and surface hyperplasia [hematoxylin and eosin (H\&E) staining; magnification, x400]. (C) Gastric mucosa pathology revealed poorly differentiated carcinoma of the gastric fundus, with a squamous epithelial component (H\&E staining; magnification, $\mathrm{x} 400)$.
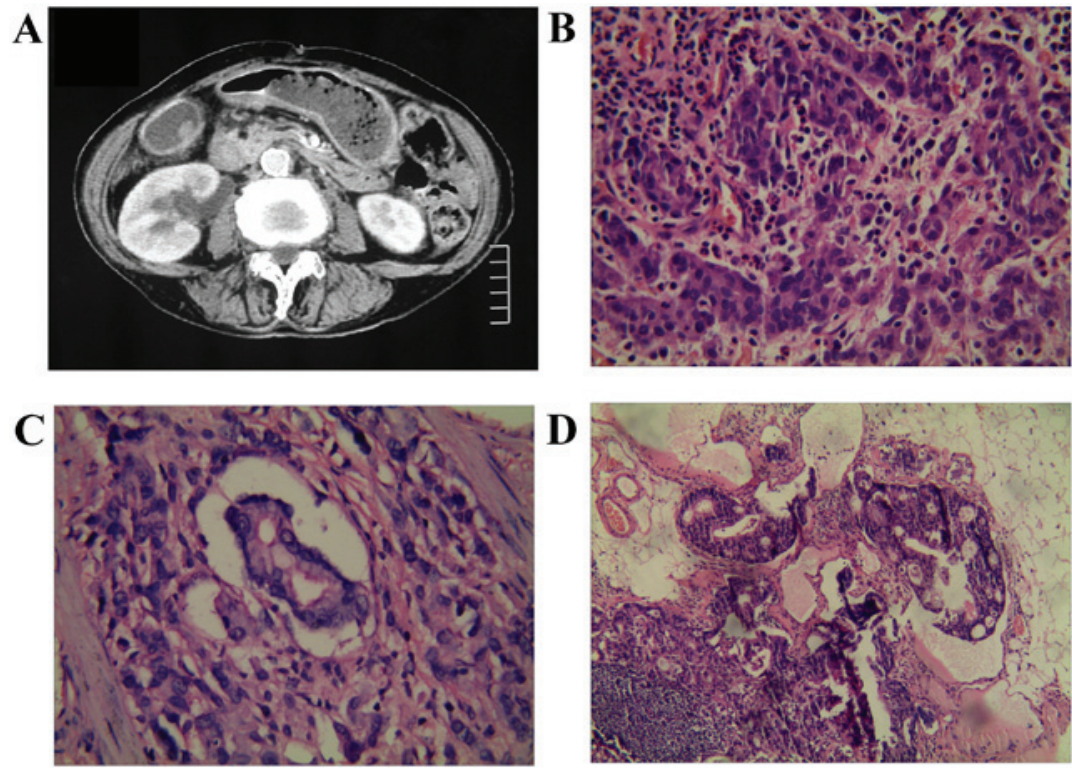

Figure 2. (A) Abdominal computed tomography scan showing a hyperechoic area located in the gallbladder. (B) Gallbladder adenocarcinoma [hematoxylin and eosin (H\&E) staining; magnification, $x$ 400]. (C) Gastric adenocarcinoma (H\&E staining; magnification, x400). (D) Intravascular gastric adenocarcinoma embolus (H\&E staining; magnification, x100).

epithelia share a common origin (the endoderm) and exhibit similar potentials of differentiation, as they are derived from the same cell lines. In addition, the gallbladder and gastric mucosae are susceptible to stimulation by mutagenic factors and, therefore, progress from intestinal metaplasia through continuous proliferation of atypical cells to precancerous lesions and, ultimately, formation of cancer foci.

The definitive causes of MPM remain unclear, but established risk factors include the following: i) Genetic factors. It was reported that $14-40 \%$ of patients with MPM have a family history or previous history of tumor(s) (9). ii) Chromosomal mutations and abnormal expression of oncogenes increase susceptibility to malignancy. Importantly, microsatellite instability (MSI) refers to substantial augmentation or heterozygosity loss of simple repetitive sequences caused by replication errors in the eukaryotic genome; these changes lead to high gene polymorphism, referred to as MSI. MSI exhibits high individual variability but stable inheritance, as well as low mutation rates in family lines. In addition, methylation of promoter region $\mathrm{CpG}$ islands causes inactivation of cancer suppressor gene transcription, inducing tumorigenesis and promoting uncontrollable tumor cell proliferation, which is also a phenotype of cytoplasmic inheritance instability $(10,11)$. Currently, it is believed that DNA mismatch repair gene defects in colorectal cancer cases are the molecular basis of primary colorectal cancer accompanied with gastric cancer. iii) Immunodeficiency and immune escape of cancer cells. A characteristic feature of cancer cells is immune escape. The internal environment is regulated via the nerve-endocrine-immune network. With increasing age, particularly during the 5th and 6th decades of life, the human immune system begins to age. For example, the thymus exhibits fibrotic changes after 40 years of age and it is eventually completely replaced by adipose tissue; thus, the monitoring function of immune cells on tumor cell is weakened and the aging of the immune system renders the internal environment more favorable for the growth and proliferation of tumor cells. iv) Application of radiotherapy, chemotherapy and relevant clinical medications. It is well known that radioactive radiation exhibits the dual function of treating as well as promoting the development of cancers. It was previously indicated that the radiological dose delivered to treat a primary cancer is sufficient to induce a secondary cancer. With the widespread application of intensity-modulated radiation therapy (12), the 
stochastic effect of high volume and small dosage increases the rate of radiation-induced cancer. In addition, agents used in tumor chemotherapy may induce disease; for example, alkylating agents induce the development of leukemia and lymphoma, while tamoxifen and others increase the occurrence rate of endometrial cancer. The carcinogenic effects of these anticancer drugs are exerted through suppressing the function of immune system, inducing lymphocyte apoptosis and, therefore, increasing susceptibility to MPM under the influence of the same carcinogenic factors. In addition, other risk factors, including host susceptibility, smoking history and gender, may be associated with occurrence of MPM.

In 1931, Warren and Gates formulated a criterion for the diagnosis of MPM (13), which was later improved to cover the following aspects: i) Each tumor must be verified as a malignancy by pathological examination; ii) each tumor must be independent, with clear boundaries and individual pathological characteristics; iii) the possibility of recurrent or metastatic cancer must be excluded; and iv) tumors develop in different sites or organs through specific metastatic channels. Clinically, when two tumors occur within 6 months, they are referred to as synchronous cancers (SCs); otherwise, they are referred to as metachronous cancers (MCs). However, one difficulty regarding the diagnosis of MPM lies with timely discovery of the second tumors. Therefore, SCs are easily missed and MCs tend to be misdiagnosed as metastatic or recurrent tumors. These conditions may affect the treatment and prognosis of MPM. If radical surgery is first conducted after a definitive diagnosis of MPM, the curative efficacy and postoperative 5-year survival do not differ from those of single malignancies. Second tumors frequently appear within 1-3 months after initial treatment. However, it is also reported that at 8.5-11 years after primary cancer surgery there is a high occurrence rate of secondary tumors, with a first peak at 4-5 years and a second peak at 10-12 years after the operation (14). However, palliative therapies, such as adjuvant radiotherapy and chemotherapy, are mainly adopted for recurrent or metastatic cancers. This treating principle also leads to a better prognosis in MPM cases compared with metastatic or recurrent cancer cases. The prognosis of MPM is closely associated with the time interval between the two cancers, site, pathological pattern and tumor stage. The shorter the time interval, the poorer the prognosis and the higher the mortality risk. In addition, the prognosis is poorer in SC cases compared with that in MC cases. It is also reported that the overall survival rates of $\mathrm{MC}$ and SC do not differ significantly. However, there is no large-scale clinical study on the survival rate of MPM. The other difficulty lies with precise differentiation between a second primary cancer and metastatic/recurrent cancer. Laboratory examinations and imaging cannot differentiate between the two groups; the differentiation mainly depends on postoperative pathological examinations. The survival of MPM patients depends on cancer foci with high-grade malignancy. Due to missed diagnosis and misdiagnosis, the majority of MPM patients succumb to the second and/or third cancers.

In the two cases reported herein, $\mathrm{LC}$ was conducted in case 1 . Due to inherent defects of laparoscopy, particularly in cases without invasion of the serosal layer, the gastric tumor was misdiagnosed during the operation. An open surgery was performed in case 2. During intraoperative exploration of the abdominal cavity, cancer foci in the fundus of the stomach were identified and the operative procedure was changed, in order to prevent misdiagnosis. Thus, the second cancer focus was identified following surgery for another cancer focus. If precise diagnosis and differentiation of multiple cancer foci may be achieved preoperatively, individualized therapeutic regimens may be implemented based on tumor stage and general patient condition, to achieve optimal outcomes. It may be concluded that, with respect to clinical diagnosis, the possibility of multiple foci should not be overlooked due to the identification of a specific focus in the patient. The detection rate of MPM is associated with the clinical experience and theoretical knowledge of the surgeon, including awareness of the possibility of MPM. In addition, explaining multiple clinical manifestations through suspicion of a single disease, based mainly on prevalence rates, is not advisable. A qualified surgeon should develop a clinical and practical experience-based scientific thinking mode, improve vigilance and reach a comprehensive diagnosis. In order to avoid missed diagnosis and misdiagnosis, X-ray radiography with double gas-barium contrast for upper gastrointestinal tract, or even positron emission tomography/CT, may be conducted preoperatively to demonstrate the metabolic activity of different tissues and determine tumor site and stage. During surgery, gastrofiberscopy and B-scan ultrasound may be used as auxiliary tools to establish the diagnosis of MPM. Postoperatively, standard pathological biopsy should be conducted to diagnose suspected foci; when necessary, immunohistochemical analysis may also be performed. During follow-up, regular re-examinations of color Doppler ultrasound, gastroscopy, CT and other imaging modalities should be used to determine the presence of MPM. Generally, re-examination should be performed every 3 months during the first year after surgery, every 6 months during the second to fifth years, and once a year thereafter.

\section{Acknowledgements}

The authors would like to thank Zheng Lu and Cong-You Gu for the pathological examination and structural editing. The present study was supported by the Tackling Key Science and Technology Project of Anhui Province, China (grant no. 1604a0802088).

\section{References}

1. Mariotto AB, Rowland JH, Ries LA, Scoppa S and Feuer EJ: Multiple cancer prevalence: A growing challenge in long-term survivorship. Cancer Epidemiol Biomarkers Prev 16: 566-571, 2007.

2. Demandante CG, Troyer DA and Miles TP: Multiple primary malignant neoplasms: Case report and a comprehensive review of the literature. Am J Clin Oncol 26: 79-83, 2003.

3. Kaneko S and Yamaguchi N: Epidemiological analysis of site relationships of synchronous and metachronous multiple primary cancers in the national cancer center, Japan, 1962-1996. Jpn J Clin OncoI 29: 96-105, 1999.

4. Bratislav T, Branimir N, Mihailo B, Jovan K, Veljović M and Dejan Z: Synchronous gastric and colonic cancer-A case report. Vojnosanit Pregl 72: 642-645, 2015.

5. Kim JH, Rha SY, Kim C, Kim GM, Yoon SH, Kim KH, Kim MJ, Ahn JB, Chung HC, Roh JK and Kim HS: Clinicopathologic features of metachronous or synchronous gastric cancer patients with three or more primary sites. Cancer Res Treat 42: 217-224, 2010. 
6. Tamura M, Shinagawa M and Funaki Y: Synchronous Triple Early Cancers Occurring in the Stomach, Colon and Gallbladder. Asian J Surg 26: 46-49, 2003.

7. Slaughter DP, Southwick HW and Smejkal W: Field cancerization in oral stratified squamous epithelium; clinical implications of multicentric origin. Cancer 6: 963-968, 1953

8. Brown SR, Finan PJ, Hall NR and Bishop DT: Incidence of DNA replication errors in patients with multiple primary cancers. Dis Colon Rectum 41: 765-769, 1998.

9. Mortel CG: Multiple primary malignant neoplasms: Historical perspectives. Cancer 40 (4 Suppl): S1786-S1792, 1977.

10. Lee SY, Kim DW, Lee HS, Ihn MH, Oh HK, Min BS, Kim WR, Huh JW, Yun JA, Lee KY, et al: Low-Level Microsatellite Instability as a Potential Prognostic Factor in Sporadic Colorectal Cancer. Medicine (Baltimore) 94: e2260, 2015.

11. Weisenberger DJ, Levine AJ, Long TI, Buchanan DD, Walters R, Clendenning M, Rosty C, Joshi AD, Stern MC, LeMarchand L, et al: Association of the colorectal $\mathrm{CpG}$ island methylator phenotype with molecular features, risk factors, and family history. Cancer Epidemiol Biomarkers Prev 24: 512-519, 2015.
12. Nakamura S, Murakami N, Inaba K, Wakita A, Kobayashi K, Takahashi K, Okamoto H, Umezawa R, Morota M, Sumi M, et al: After low and high dose-rate interstitial brachytherapy followed by IMRT radiotherapy for intermediate and high risk prostate cancer. BMC Cance 16: 296, 2016.

13. Warren S and Gates O: Multiple primary malignant tumors: A survey of the literature and a statistical study. Am J Cancer 16: 1358-1414, 1932

14. Lan YT, Lin JK, Li AF, Lin TC, Chen WS, Jiang JK, Yang SH, Wang HS and Chang SC: Metachronous colorectal cancer: Necessity of post-operative colonoscopic surveillance. Int J Colorectal Dis 20: 121-125, 2005. 